Pacific Journal of Mathematics

STABLE PARALLELIZABILITY OF PARTIALLY ORIENTED PARATEWARA SANKA RAN AND PETER ZVENGRows 


\title{
STABLE PARALLELIZABILITY OF PARTIALLY ORIENTED FLAG MANIFOLDS
}

\section{P. SANKARAN AND P. ZVENGROWSKI}

\begin{abstract}
This paper solves the questions of stable parallelizability and parallelizability for the family of partially oriented (p.o.) flag manifolds, except for a few undecided cases. In particular, for the oriented Grassmannians $\tilde{G}_{k}\left(\mathbf{R}^{n}\right)$ it is proved that apart from the spheres $S^{1}, S^{3}$, and $S^{7}$ only $\tilde{G}_{3}\left(\mathbf{R}^{6}\right)$ is parallelizable, and only $\tilde{G}_{2}\left(\mathbf{R}^{4}\right)$ is stably parallelizable and not parallelizable. Negative results are derived for the most part using $\mathrm{KO}$ theory and the "inclusion method", while positive results are mainly based on the " $\lambda{ }^{2}$ construction".
\end{abstract}

1. Introduction. The p.o. flag manifolds are real flag manifolds with additional structure of orientations on some of the orthogonal subspaces constituting each flag. More precisely the p.o. flag manifold $G\left(n_{1}, \ldots, n_{r} \mid n_{r+1}, \ldots, n_{s}\right)$ is the space of all mutually orthogonal subspaces $\sigma_{1}, \ldots, \sigma_{s}$ of $\mathbf{R}^{n}$, where $n=n_{1}+\cdots+n_{s}, \operatorname{dim} \sigma_{i}=n_{i}$, and $\sigma_{1}, \ldots, \sigma_{r}$ are oriented. The number " $s$ " will always be used for the "length" of the flag. Familiar examples are $G(1, \ldots, 1 \mid n-r)=V_{n, r}$, the real Stiefel manifold of orthonormal $r$-frames in $\mathbf{R}^{n}$, while $G(k, n-k \mid)=\tilde{G}_{k}\left(\mathbf{R}^{n}\right)$, the Grassmann manifold of oriented $k$-planes in $\mathbf{R}^{n}$. The stable parallelizability or parallelizability of these manifolds is a natural question, going back to work of Kervaire and Milnor in 1958 for $S^{n-1}=V_{n, 1}$, and Sutherland in 1964 for $V_{n, r}, r>1$. More recently I. D. Miatello and R. J. Miatello (1982) and R. Stong (1984) have studied this question. Here we settle the stable parallelizability of p.o. flag manifolds, apart from a few unsolved cases, and, among those known to be stably parallelizable, completely determine which are parallelizable.

The corresponding problem for flag manifolds $(r=0)$ was settled in 1984 by Korbaš [12] and Sankaran-Zvengrowski [18] (the latter also covering the complex and quaternionic flag manifolds). We therefore consider only the case $r \geq 1$ here. Notice that there is an obvious $2^{r}$-fold covering map

$$
G\left(n_{1}, \ldots, n_{r} \mid n_{r+1}, \ldots, n_{s}\right) \rightarrow G\left(n_{1}, \ldots, n_{s}\right),
$$

and both spaces have the same dimension. In case $r=s$ we make the additional convention that the orientations on $\sigma_{1}, \ldots, \sigma_{s}$ induce the standard orientation on $\mathbf{R}^{n}=\sigma_{1} \oplus \cdots \oplus \sigma_{s}$. With this convention 
$G\left(n_{1}, \ldots, n_{s-1} \mid n_{s}\right)=G\left(n_{1}, \ldots, n_{s} \mid\right)$, and we write simply $\tilde{G}\left(n_{1}, \ldots, n_{s}\right)$ here. Our main results are now summarized in 1.1 and 1.2. We consider only p.o. flag manifolds $G\left(n_{1}, \ldots, n_{r} \mid n_{r+1}, \ldots, n_{s}\right)$ with $n_{1} \geq \cdots \geq n_{r}$ and $n_{r+1} \geq \cdots \geq n_{s}$ since

$$
G\left(n_{1}, \ldots, n_{r} \mid n_{r+1}, \ldots, n_{s}\right) \cong G\left(m_{1}, \ldots, m_{r} \mid m_{r+1}, \ldots, m_{s}\right)
$$

if $m_{1}, \ldots, m_{r}$ and $m_{r+1}, \ldots, m_{s}$ are rearrangements of $n_{1}, \ldots, n_{r}$ and $n_{r+1}, \ldots, n_{s}$ respectively.

1.1. TheOREM. (A) For $s=2, k \neq 1, n-1, \tilde{G}_{k}\left(\mathbf{R}^{n}\right)$ is stably parallelizable if and only if $(n, k)=(4,2)$ or $(6,3)$, and only $\tilde{G}_{3}\left(\mathbf{R}^{6}\right)$ is parallelizable (the cases $k=1, n-1$ are well known and omitted here).

Now for (B), (C) and (D) we assume $s \geq 3$.

(B) Assume that at least two of the numbers $n_{1}, \ldots, n_{s}$ are greater than 1. Then $G\left(n_{1}, \ldots, n_{r} \mid n_{r+1}, \ldots, n_{s}\right)$ is not stably parallelizable if $\left\{n_{1}, \ldots, n_{s}\right\} \not \subset\{3,1\}$ or $\{2,1\}$.

(C) Consider $G\left(n_{1}, \ldots, n_{r} \mid n_{r+1}, \ldots, n_{s}\right)$ with $1 \leq r<s-1$, and either $n_{q}>1$ for precisely one $q$, or $\left\{n_{1}, \ldots, n_{s}\right\}=\{3,1\}$, or $\left\{n_{1}, \ldots, n_{s}\right\}=$ $\{2,1\}$ with $n_{r+1}=2$. Then $G\left(n_{1}, \ldots, n_{r} \mid n_{r+1}, \ldots, n_{s}\right)$ is not stably parallelizable in the following cases.

(i) Let $n_{r+1}=\cdots=n_{s}=1$.

(a) $r \leq s-2, n_{i} \neq 1,2,6$ for some $i \leq r$.

(b) $r \leq s-4, n_{l} \neq 1,2$ for some $i \leq r$.

(ii) $n_{r+1}>1, r<s-2$.

(iii) $r=s-2, n_{s-1}>1,\left(n_{1}, n_{s-1}, n_{s}\right) \neq(1,3,1),(1,7,1)$.

(D) The following p.o. flag manifolds are stably parallelizable.

(i) $\tilde{G}(3, \ldots, 3,1, \ldots, 1), l \geq 0$.

(ii) $G(2, \ldots, 2 \mid 1, \ldots, 1), l \geq 0$

(iii) $G(6 \mid \stackrel{(k)}{1}, 1), G(6,1 \mid 1,1)$.

of these only $\tilde{G}(2, \ldots, 2)$ and $\tilde{G}(2, \ldots, 2,1)$ are not parallelizable.

Note: The parallelizability of $G(1, \ldots, k, 1 \mid 1, \ldots, 1)$ is immediate from that of $G(1, \ldots, 1)$, proved in [18].

Theorem 1.1 leaves a few unsolved cases and these are now listed.

1.2. Unsolved cases. (for stable parallelizability)

$$
\begin{array}{ll}
G(1, \ldots, 1 \mid 3,1), & s \geq 3 \\
G(1, \ldots, 1 \mid 7,1), & s \geq 3 \\
G(6,1, \ldots, 1 \mid 1,1), & s \geq 5 \\
G(6,1, \ldots, 1 \mid 1,1,1), & s \geq 4
\end{array}
$$


The families of flag and p.o. flag manifolds were first studied in full generality by Lam [13], who derived the formula for the tangent bundle

$$
\tau G\left(n_{1}, \ldots, n_{s}\right) \approx \sum_{i<j} \xi_{i} \otimes \xi_{j},
$$

where $\xi_{i}$ is the vector bundle over $G\left(n_{1}, \ldots, n_{s}\right)$ whose fibre at $\left(\sigma_{1}, \ldots, \sigma_{s}\right)$ is the $n_{i}$-dimensional vector space $\sigma_{i}$. Notice that $\xi_{1} \oplus \cdots \oplus \xi_{s} \approx n \varepsilon$, the trivial $n$-dimensional bundle, and that Lam's formula holds for $G\left(n_{1}, \ldots, n_{r} \mid n_{r+1}, \ldots, n_{s}\right)$ as well, where $\xi_{1}, \ldots, \xi_{r}$ are now oriented vector bundles.

In $\$ 2$ the case $s=2$, i.e. the oriented Grassmannians, is solved (Theorem 1.1A). This case is also the subject of [15], but there is a gap in their proof (p. 350, line 1). We therefore give most of the details of our proof, which in any case is shorter. Positive results on stable parallelizability are obtained in $\S 3$, using a generalized $\lambda^{2}$ (second exterior power) construction. Negative results are obtained in $\$ 4$ using the "inclusion" method as in [18] and other ad hoc techniques. Recall the isomorphisms

1.3. $\lambda^{2}(\xi \oplus \eta) \approx \lambda^{2} \xi \oplus \xi \otimes \eta \oplus \lambda^{2} \eta$, for any real vector bundles $\xi, \eta$,

1.4. $\lambda^{p}(\xi) \approx \lambda^{n-p}(\xi)$ for any real $n$-dimensional oriented vector bundle $\xi$ (by Hodge duality).

Another observation that will be of use in the sequel is the relation

$$
G(n-2 \mid 1,1) \cong X_{n, 2}
$$

with projective Stiefel manifolds. The homeomorphism is given by $\varphi\left(\sigma_{1}, \sigma_{2}, \sigma_{3}\right)=\left[\begin{array}{c}a \\ b\end{array}\right]$, where $a, b$ are any unit vectors in $\sigma_{2}, \sigma_{3}$ respectively such that $\sigma_{1}, a, b$ induces the usual orientation on $\mathbf{R}^{n}$ (note $\sigma_{1}$ is already oriented), and $\left[\begin{array}{c}a \\ b\end{array}\right]=\left[\begin{array}{c}-a \\ -b\end{array}\right] \in X_{n, 2}$.

2. The stable parallelizability of $\tilde{G}_{k}\left(\mathbf{R}^{n}\right)$. In this section we prove Theorem 1.1(A).

Note that in case $k=1$ or $n-1, \tilde{G}_{k}\left(\mathbf{R}^{n}\right) \cong S^{n-1}$ and the solution for their parallelizability was obtained independently by Kervaire [11] and Milnor [16]. $\beta=\tilde{\beta}_{n, k}$.

We identify $\tilde{G}_{k}\left(\mathbf{R}^{n}\right)$ with $\tilde{G}(k, n-k)$ and write $\xi_{1}=\gamma=\tilde{\gamma}_{n, k}, \xi_{2}=$

We have $\gamma \oplus \beta \approx n \varepsilon$ and $\tau=\tau\left(\tilde{G}_{n, k}\right) \approx \gamma \otimes \beta$. We apply the functor $\lambda^{2}$ and 1.3 to the relation $n \varepsilon \approx \gamma \oplus \beta$ to obtain

$$
\begin{aligned}
\left(\begin{array}{l}
n \\
2
\end{array}\right) \varepsilon & \approx \lambda^{2}(\gamma \oplus \beta) \approx \lambda^{2}(\gamma) \oplus \lambda^{2}(\beta) \oplus \gamma \otimes \beta \\
& \approx \lambda^{2}(\gamma) \oplus \lambda^{2}(\beta) \oplus \tau .
\end{aligned}
$$


When $n=4, k=2=n-2$. Thus by $1.4 \lambda^{2}(\gamma) \approx \varepsilon \approx \lambda^{2}(\beta)$. Therefore 2.1.1 gives $6 \varepsilon \approx 2 \varepsilon \oplus \tau$, showing that $\tilde{G}_{2}\left(\mathbf{R}^{4}\right)$ is stably parallelizable. The nonparallelizability now follows from the fact that $\operatorname{span} \tilde{G}_{2}\left(\mathbf{R}^{4}\right)=0$ as $\chi\left(\tilde{G}_{2}\left(\mathbf{R}^{4}\right)\right)=2 \chi\left(G_{2}\left(\mathbf{R}^{4}\right)\right) \neq 0$ (cf. Lemma $\left.2.3[9]\right)$.

When $n=5, k=2$ or 3 , and since $\tilde{G}_{2}\left(\mathbf{R}^{5}\right) \cong \tilde{G}_{3}\left(\mathbf{R}^{5}\right)$ we need only consider the case $k=2$. We proceed as before and obtain from (2.1.1) and 1.4 the relation $\left(\begin{array}{l}5 \\ 2\end{array}\right) \varepsilon \approx \varepsilon \oplus \beta \oplus \tau$. Thus $\tau \sim \gamma+0$ in $\tilde{\mathrm{K}} \mathrm{O}\left(\tilde{G}_{2}\left(\mathbf{R}^{5}\right)\right)$. In other words $\tilde{G}_{2}\left(\mathbf{R}^{5}\right)$ is not stably parallelizable.

Let $n=6, k=3$. Again by the same arguments one shows that

$$
\left(\begin{array}{l}
6 \\
2
\end{array}\right) \varepsilon \approx \lambda^{2}(\gamma) \oplus \lambda^{2}(\beta) \oplus \tau \approx \gamma \oplus \beta \oplus \tau \approx 6 \varepsilon \oplus \tau .
$$

Hence $\tilde{G}_{3}\left(\mathbf{R}^{6}\right)$ is stably parallelizable.

We know from the work of Leite and Miatello [14] that span $\tilde{G}_{3}\left(\mathbf{R}^{6}\right)>1=\operatorname{span} S^{9}$. Since $\operatorname{dim} \tilde{G}_{3}\left(\mathbf{R}^{6}\right)=9$, by the Bredon-Kosinski Theorem [7] we conclude that $\tilde{G}_{3}\left(\mathbf{R}^{6}\right)$ is parallelizable.

Now only the cases $\tilde{G}_{k}\left(\mathbf{R}^{n}\right)$ with $n \geq 6,(n, k) \neq(6,3)$ need to be considered. Without loss of generality we assume that $2 k \leq n$. It follows that $n-k \geq 4=\operatorname{dim} \mathbf{C} P^{2}$. Thus every real (orientable) $k$-plane bundle $\eta$ over $\mathbf{C} P^{2}$ can be classified by a map $g: \mathbf{C} P^{2} \rightarrow \tilde{G}_{k}\left(\mathbf{R}^{n}\right)$ so that $\eta \approx g^{*}(\gamma)$ (cf. §19, [19]). Taking $\eta=\xi \oplus(k-2) \varepsilon$ where $\xi$ is the underlying real 2-plane bundle of the canonical complex line bundle over $\mathbf{C} P^{2}$ we obtain the following equalities in $\mathrm{KO}\left(\mathrm{CP}^{2}\right)$ :

$$
\begin{aligned}
g^{*}(\gamma) & \approx \xi \oplus(k-2) \varepsilon \\
g^{*}(\beta) & \approx g^{*}(n \varepsilon-\gamma), \\
& \approx(n-k+2) \varepsilon-\xi
\end{aligned}
$$

Thus

$$
\begin{aligned}
g^{*}(\tau) & \approx g^{*}(\gamma \otimes \beta) \approx g^{*}(\gamma) \otimes g^{*}(\beta) \\
& \approx(\xi \oplus(k-2) \varepsilon) \otimes((n-k+2) \varepsilon-\xi) \\
& \approx(n-2 k+4) \xi \oplus m \varepsilon-\xi \otimes \xi
\end{aligned}
$$

for a suitable $m$. Using the relation $\xi \otimes \xi \approx 4 \xi-4 \varepsilon$ in $\mathrm{KO}\left(\mathrm{C} P^{2}\right)$ and the fact that $\xi$ has infinite order (cf. Fujii [8]) we obtain the stable equivalence $g^{*}(\tau) \sim(n-2 k) \xi+0$ for $n \neq 2 k$. Thus $\tilde{G}_{k}\left(\mathbf{R}^{n}\right)$ is not stably parallalizable for $n \neq 2 k$.

In case $n=2 k, k \geq 4$, consider the inclusion $\mathbf{R}^{8} \rightarrow \mathbf{R}^{k-4} \oplus \mathbf{R}^{8} \oplus$ $\mathbf{R}^{k-4}$. This induces an inclusion $j: \tilde{G}_{4}\left(\mathbf{R}^{8}\right) \rightarrow \tilde{G}_{k}\left(\mathbf{R}^{2 k}\right)$ where $j(A)=\tilde{X}+$ $\tilde{A}, \quad \tilde{X}=\mathbf{R}^{k-4} \oplus 0 \oplus 0$, and $\tilde{A}=0 \oplus A \oplus 0$. It is readily seen that 


$$
\begin{aligned}
& j^{*}\left(\tilde{\gamma}_{2 k, k}\right)=\tilde{\gamma}_{8,4} \oplus(k-4) \varepsilon \text {. Hence } \\
& j^{*}\left(\tau\left(\tilde{G}_{k}\left(\mathbf{R}^{2 k}\right)\right)\right) \approx j^{*}\left(\tilde{\gamma}_{2 k, k} \otimes \tilde{\beta}_{2 k, k}\right) \\
& \approx j^{*}\left(\tilde{\gamma}_{2 k, k}\right) \otimes j^{*}\left(\tilde{\beta}_{2 k, k}\right) \\
& \approx\left(\tilde{\gamma}_{8,4} \oplus(k-4) \varepsilon\right) \otimes\left(\tilde{\beta}_{8,4} \oplus(k-4) \varepsilon\right) \\
& \approx \tilde{\gamma}_{8,4} \otimes \tilde{\beta}_{8,4} \oplus(k-4) \varepsilon \otimes\left(\tilde{\beta}_{8,4} \oplus \tilde{\gamma}_{8,4}\right) \oplus(k-4)^{2} \varepsilon \\
& \approx \tau\left(\tilde{G}_{4}\left(\mathbf{R}^{8}\right)\right) \oplus(k-4) \varepsilon \otimes 8 \varepsilon \oplus(k-4)^{2} \varepsilon \\
& \sim \tau\left(\tilde{G}_{4}\left(\mathbf{R}^{8}\right)\right) \text {. }
\end{aligned}
$$

Thus to prove that $\tilde{G}_{k}\left(\mathbf{R}^{2 k}\right), k \geq 4$, is not stably parallelizable, it suffices to show that $\tau\left(\tilde{G}_{4}\left(\mathbf{R}^{8}\right)\right) \times 0$. V. Bartík and J. Korbaš [4] have computed $w_{i}\left(G_{k}\left(\mathbf{R}^{n}\right)\right)$ for $1 \leq i \leq 9$. From their results $w_{8}\left(G_{4}\left(\mathbf{R}^{8}\right)\right)=w_{2}^{4}+w_{1}^{2} w_{3}^{2} \in$ $H^{8}\left(G_{4}\left(\mathbf{R}^{8}\right) ; \mathbf{Z}_{2}\right)$ where $w_{i}=w_{i}\left(\gamma_{8,4}\right)$. It follows that $w_{8}\left(\tilde{G}_{4}\left(\mathbf{R}^{8}\right)\right)=$ $\left(w_{2}\left(\tilde{\gamma}_{8,4}\right)\right)^{4} \in H^{8}\left(\tilde{G}_{4}\left(\mathbf{R}^{8}\right) ; \mathbf{Z}_{2}\right)$. One uses the Gysin sequence associated to the double covering $\tilde{G}_{4}\left(\mathbf{R}^{8}\right) \rightarrow G_{4}\left(\mathbf{R}^{8}\right)$ together with the known cohomology of $G_{4}\left(\mathbf{R}^{8}\right)$ to establish that $\left(w_{2}\left(\tilde{\gamma}_{8,4}\right)\right)^{4} \neq 0$ in $H^{8}\left(\tilde{G}_{4}\left(\mathbf{R}^{8}\right) ; \mathbf{Z}_{2}\right)$. This completes the proof.

REMARK. The authors could find no way to handle the exceptional case $\tilde{G}_{4}\left(\mathbf{R}^{8}\right)$ without the use of Stiefel-Whitney classes. Other methods of calculating $w\left(\tilde{G}_{4}\left(\mathbf{R}^{8}\right)\right)$ can be found in [17] or in [15].

3. The generalized $\lambda^{2}$-construction. Using the so-called $\lambda^{2}$-construction, we prove Theorem 1.1(D).

In cases (i) and (ii) we apply the $\lambda^{2}$ functor to the following bundle isomorphism

$$
n \varepsilon \approx \xi_{1} \oplus \cdots \oplus \xi_{s}
$$

to obtain

$$
\begin{aligned}
\left(\begin{array}{l}
n \\
2
\end{array}\right) \varepsilon & \approx \lambda^{2}(n \varepsilon) \approx \lambda^{2}\left(\xi_{1} \oplus \cdots \oplus \xi_{s}\right) \\
& \approx \sum_{1 \leq j \leq s} \lambda^{2}\left(\xi_{j}\right) \oplus\left(\sum_{1 \leq i<j \leq s} \xi_{i} \otimes \xi_{j}\right) \\
& \approx \sum_{1 \leq j \leq s} \lambda^{2}\left(\xi_{j}\right) \oplus \tau .
\end{aligned}
$$


We now use 1.4 and the fact that $\lambda^{2}(\zeta) \approx 0$ for a line bundle $\zeta$ to simplify the right hand side. Since from 3.1.1 one has the stable equivalence $\xi_{1} \oplus \cdots \oplus \xi_{k} \sim 3 k \varepsilon$ in case (i), we obtain

$$
\left(\begin{array}{l}
n \\
2
\end{array}\right) \varepsilon \sim m \varepsilon \oplus \tau
$$

where $m=3 k$ in case (i), and $m=k$ in case (ii). This proves stable parallelizability in cases (i) and (ii). The proof of parallelizability in cases (i) and (ii) is postposed to the end of this section.

Parallelizability of $G(6 \mid 1,1) \cong X_{8,2}$ (cf. 1.5 ) is due to Zvengrowski [23]. We now show that $M=G(6,1 \mid 1,1)$ is parallelizable. We have $\xi_{2} \approx \varepsilon$. Since $\Sigma_{1 \leq i \leq 4} \xi_{i} \approx 9 \varepsilon$, and since $\xi_{1}$ and $\xi_{2}$ are oriented, it follows that $w_{1}\left(\xi_{3}\right)=w_{1}\left(\xi_{4}\right)$. Since a line bundle is determined by its first Stiefel-Whitney class, we must have $\xi_{3} \approx \xi_{4}$. Call this line bundle $\zeta$. Now using the fact that $\xi \otimes \varepsilon \approx \varepsilon \otimes \xi \approx \xi$ we have the following bundle isomorphisms

$$
\begin{aligned}
\tau & \approx \sum_{1 \leq i<j \leq 4} \xi_{i} \otimes \xi_{j} \\
& \approx \xi_{1} \oplus \xi_{3} \oplus \xi_{4} \oplus \xi_{1} \otimes\left(\xi_{3} \oplus \xi_{4}\right) \oplus \xi_{3} \otimes \xi_{4} \\
& \approx \xi_{1} \oplus \xi_{3} \oplus \xi_{4} \oplus \xi_{1} \otimes 2 \zeta \oplus \xi_{2}
\end{aligned}
$$

as $\xi_{3} \otimes \xi_{4} \approx \zeta \otimes \zeta \approx \varepsilon \approx \xi_{2}$. Hence

$$
\tau \approx \xi_{1} \oplus \xi_{2} \oplus \xi_{3} \oplus \xi_{4} \oplus \xi_{1} \otimes 2 \zeta \approx 9 \varepsilon \oplus \xi_{1} \otimes 2 \zeta,
$$

showing that $\operatorname{span} M \geq 9>\operatorname{span} S^{m}, m=\operatorname{dim} M=21$. We wish to apply the Bredon-Kosinski Theorem to prove that $M$ is parallelizable. Thus it remains to prove that $\tau \sim 0$. In $\mathrm{KO}(M), \xi_{1} \approx 9 \varepsilon-\xi_{2}-\xi_{3}-\xi_{4}$ $\approx 8 \varepsilon-2 \zeta$, so $\xi_{1} \otimes 2 \zeta \approx(8 \varepsilon-2 \zeta) \otimes 2 \zeta \approx 16 \zeta-4(\zeta \otimes \zeta) \approx 16 \zeta-4 \varepsilon$. Therefore in $\tilde{\mathrm{K} O}(M), \tau \sim 16 \zeta$.

Now consider the fibre map $q: M=G(6,1 \mid 1,1) \rightarrow G(8,1)=\mathbf{R} P^{8}$. Denoting the canonical line bundle over $\mathbf{R} P^{8}$ by $\xi$, we see that $q^{*}(\xi) \approx \xi_{4}$ $=\zeta$. Since the order of $\xi$ is 16 by [1], it follows that $16 \zeta \sim 0$. Hence $M=G(6,1 \mid 1,1)$ is parallelizable.

Parallelizability in case (i) follows from the work of [15] and [20]. Using a similar argument one can show that the manifolds listed in (ii) except for $M_{1}=G(2, \ldots, 2 \mid 1)$ and $M_{2}=\tilde{G}(2, \ldots, 2)$ are all parallelizable. However, we give a direct proof using $\lambda^{2}$ again in the proposition that follows. The non-parallelizability of $M_{1}$ and $M_{2}$ follows from the fact that their Euler characteristics are positive (cf. [12]) and hence their span must be zero. 
We now turn to the parallelizability proof for case (ii).

Let $m=\operatorname{dim} M=p-k$, where $p=\left(\begin{array}{c}n \\ 2\end{array}\right)$. Regard $\mathbf{R}^{P}$ as $\lambda^{2}\left(\mathbf{R}^{n}\right)$. Define a map $g: M \rightarrow G(1, \ldots, 1 \mid m) \cong V_{p, k}$ as follows: For an oriented 2-plane $\sigma \subset \mathbf{R}^{n}, \lambda^{2}(\sigma)$ is an oriented line in $\lambda^{2}\left(\mathbf{R}^{n}\right)$, the orientation on it being given by the vector $a \wedge b$ where $a, b$ is any positively oriented basis of $\sigma$. Further if $\sigma \perp \sigma^{\prime}$ in $\mathbf{R}^{n}$ then $\lambda^{2}(\sigma) \perp \lambda^{2}\left(\sigma^{\prime}\right)$ in $\lambda^{2}\left(\mathbf{R}^{n}\right)$. Thus we may define $g(\underline{\sigma})=\left(\lambda^{2}\left(\sigma_{1}\right), \ldots, \lambda^{2}\left(\sigma_{k}\right), U \underline{\sigma}\right) \in G(1, \ldots, 1 \mid m)$ for $\underline{\sigma}=$ $\left(\sigma_{1}, \ldots, \sigma_{s}\right) \in M$, where

$$
U \underline{\boldsymbol{\sigma}}=\left(\lambda^{2}\left(\sigma_{1}\right)+\cdots+\lambda^{2}\left(\sigma_{k}\right)\right)^{\perp} \text { in } \lambda^{2}\left(\mathbf{R}^{n}\right) .
$$

ClaIM. $\tau M \approx g^{*}\left(\xi_{k+1}(1, \ldots, 1 \mid m)\right)$. We construct a specific bundle isomorphism $f: \tau M \rightarrow g^{*}\left(\xi_{k+1}(1, \ldots, 1 \mid m)\right)$ as follows. Let $\underline{\sigma}=$ $\left(\sigma_{1}, \ldots, \sigma_{s}\right) \in M$. The tangent space $T \underline{\sigma}$ of $M$ at $\underline{\sigma}$ is $\sum_{1 \leq i<j \leq s} \sigma_{i} \otimes \sigma_{j}$. Thus any $v \in T \underline{\sigma}$ may be expressed as a sum of terms having the form $v_{1} \otimes v_{2}+\cdots+v_{s-1} \otimes v_{s}$ with $v_{j} \in \sigma_{j}$. It is easy to check that $\sum_{1 \leq i<j \leq s} v_{i} \wedge v_{j} \in U_{\underline{\sigma}}$. Now $f$ is defined by

$$
f\left(\underline{\sigma}, \sum_{1 \leq i<j \leq s} v_{i} \otimes v_{j}\right)=\left(\underline{\sigma},\left(g(\underline{\sigma}), \sum_{1 \leq i<j \leq s} v_{i} \wedge v_{j}\right)\right) \text {. }
$$

Continuity of $f$ is obvious. Fibres are preserved by $f$ and, restricted to each fibre, it is a linear isomorphism since the kernel of the homomorphism $\mathbf{R}^{n} \otimes \mathbf{R}^{n} \rightarrow \lambda^{2}\left(\mathbf{R}^{n}\right)$ intersects $\sum_{1 \leq i<j \leq s} \sigma_{i} \otimes \sigma_{j}$ only in 0. Hence $\tau M \approx g^{*}\left(\xi_{k+1}(1, \ldots, 1 \mid m)\right)$.

To complete the proof we now show that $g$ is null-homotopic (cf. Theorem $4.7 \mathrm{Ch}$. II [10]). Since $g$ depends only on $\sigma_{1}, \ldots, \sigma_{k}$ it can be factored as follows:

$$
\begin{array}{ccc}
M & \stackrel{g}{\rightarrow} & V_{p, k} \\
\pi \downarrow & & \uparrow g^{\prime} \\
M^{\prime}= & &
\end{array}
$$

where $\pi(\underline{\sigma})=\left(\sigma_{1}, \ldots, \sigma_{k}, \sigma_{k+1}+\cdots+\sigma_{s}\right)$ for $\underline{\sigma} \in M$, and $g^{\prime}$ is the map induced by $g$. Now

$$
\begin{aligned}
\operatorname{dim} M^{\prime} & =p-k-\left(\begin{array}{l}
l \\
2
\end{array}\right) \leq p-k-1 \text { for } l \geq 2 \\
& =\text { connectivity of } V_{p, k} .
\end{aligned}
$$

Hence $g^{\prime}$ is null-homotopic. Therefore $g=g^{\prime} \circ \pi$ is also null-homotopic, completing the proof. 
4. Results for partially oriented flag manifolds. In this section we complete the proof of 1.1(B) and 1.1(C). We assume that $s \geq 3$, the case $s=2$ having been dealt with in $\$ 2$. In case $r=s$ we assume that $n_{2}>1$, since $\tilde{G}\left(n_{1}, 1, \ldots, 1\right) \cong V_{n, s-1}(s-1 \geq 2)$ are all known to be parallelizable [21], [13], [23]. Also we assume that $n_{1}>1$ or $n_{r+1}>1$ since $G(1, \ldots, 1)$ is parallelizable [18].

Proof of 1.1(B). Using a method similar to the one used in [18], one can show that for a suitable "inclusion"

$$
\tilde{G}_{n_{i}}\left(\mathbf{R}^{n_{i}+n_{j}}\right) \cong \tilde{G}\left(n_{i}, n_{j}\right) \stackrel{k}{\rightarrow} \tilde{G}\left(n_{1}, \ldots, n_{s}\right)
$$

the normal bundle to the imbedding $k$ is trivial. Hence $\tilde{G}\left(n_{1}, \ldots, n_{s}\right)$ is not stably parallelizable if $\tilde{G}\left(n_{i}, n_{j}\right)$ is not stably parallelizable. Our assumptions on $n_{1}, \ldots, n_{s}$ and Theorem 1.1(A) show that there exist $i$ and $j$ for which $\tilde{G}\left(n_{i}, n_{j}\right)$ is not stably parallelizable, completing the proof in the case $r=s$.

The case $r<s-1$ follows from the above and the observation that $\tilde{G}\left(n_{1}, \ldots, n_{s}\right)$ is a covering space of $G\left(n_{1}, \ldots, n_{r} \mid n_{r+1}, \ldots, n_{s}\right)$.

Note that Theorem 1.1(B) and 1.1(D) give complete results on stable parallelizability of $\tilde{G}\left(n_{1}, \ldots, n_{s}\right)$. We now turn to the proof of 1.1(C).

Recall that only the p.o. flag manifolds $G\left(n_{1}, \ldots, n_{r} \mid n_{r+1}, \ldots, n_{s}\right)$ with $1 \leq r<s-1$, and either $n_{q}>1$ for precisely one $q$, or $\left\{n_{1}, \ldots, n_{s}\right\}$ $=\{3,1\}$, or $\left\{n_{1}, \ldots, n_{s}\right\}=\{2,1\}$ with $n_{r+1}=2$ need to be considered here.

(i) (a) In this case one considers the "inclusion" of

$$
G\left(n_{i} \mid 1,1\right) \stackrel{j_{1}}{\rightarrow} G\left(n_{1}, \ldots, n_{r} \mid n_{r+1}, \ldots, n_{s}\right)
$$

which has trivial normal bundle. Now $G\left(n_{i} \mid 1,1\right) \cong X_{n_{t}+2,2}$ is not stably parallelizable since $n_{i} \neq 1,2,6$ (cf. [2]). Therefore $G\left(n_{1}, \ldots, n_{r} \mid n_{r+1}, \ldots, n_{s}\right)$ is not stably parallelizable.

(i) (b) In this case we may assume from what has been shown in (i)(a) that $n_{i}=n_{q}=6$. Now one has an inclusion

$$
j_{2}: G(6 \mid 1,1,1,1) \rightarrow G\left(n_{1}, \ldots, n_{r} \mid n_{r+1}, \ldots, n_{s}\right)
$$

with trivial normal bundle. So it suffices to show that $G(6 \mid 1,1,1,1)$ is not stably parallelizable. Indeed, consider the covering projection $f: \quad V_{10,4} \rightarrow G(6 \mid 1,1,1,1)$ which maps $\left(v_{1}, v_{2}, v_{3}, v_{4}\right) \in V_{10,4}$ to $\left(A, \mathbf{R} v_{1}, \mathbf{R} v_{2}, \mathbf{R} v_{3}, \mathbf{R} v_{4}\right)$ where $A=\left\{v_{1}, v_{2}, v_{3}, v_{4}\right\}^{\perp}$. The orientation on $A$ is given by any ordered basis $u_{1}, \ldots, u_{6}$ where $u_{1}, \ldots, u_{6}, v_{1}, v_{2}, v_{3}, v_{4}$ 
(equivalently $u_{1}, \ldots, u_{6},-v_{1},-v_{2},-v_{3},-v_{4}$ ) is in the standard orientation on $\mathbf{R}^{10}$. It is readily seen that $f$ can be factored as in the diagram below.

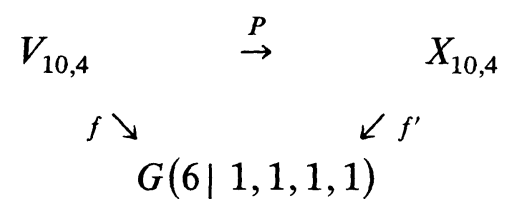

Since $f^{\prime}$ is a covering projection and since $X_{10,4}$ is not stably parallelizable (cf. [2], [3]) it follows that $G(6 \mid 1,1,1,1)$ is not stably parallelizable.

(ii) In this case one considers the inclusion $j: G\left(n_{r+1}, \ldots, n_{s}\right) \rightarrow$ $G\left(n_{1}, \ldots, n_{r} \mid n_{r+1}, \ldots, n_{s}\right)$ and uses Theorem 1 of $[18]$.

(iii) If $\left(n_{s-1}, n_{s}\right) \neq(3,1),(7,1)$ then by considering the inclusion $j: G\left(n_{s-1}, n_{s}\right) \rightarrow G\left(n_{1}, \ldots, n_{s-2} \mid n_{s-1}, n_{s}\right)$ and the negative results about the stable parallelizability of Grassmann manifolds (cf. [22]) we see that $G\left(n_{1}, \ldots, n_{s-2} \mid n_{s-1}, n_{s}\right)$ is not stably parallelizable. Finally, it remains to show that for $k \geq 1, G(3, \ldots, k, 3,1, \ldots, 1 \mid 3,1)$ is not stably parallelizable. Once again, by the inclusion method it suffices to show that $G(3 \mid 3,1)$ is not stably parallelizable. In this case we resort to the standard Stiefel-Whitney class argument as follows.

As in (3.1.2) one has $\left(\begin{array}{c}7 \\ 2\end{array}\right) \varepsilon \approx \lambda^{2}\left(\xi_{1}\right) \oplus \lambda^{2}\left(\xi_{2}\right) \oplus \lambda^{2}\left(\xi_{3}\right) \oplus \tau \approx \xi_{1} \oplus$ $\lambda^{2}\left(\xi_{2}\right) \oplus \tau$. We will prove that $w\left(\xi_{1}\right) \cdot w\left(\lambda^{2}\left(\xi_{2}\right)\right) \neq 1$. For a 3-plane bundle $\xi, w\left(\lambda^{2}(\xi)\right)=1+\left(w_{1}(\xi)\right)^{2}+w_{2}(\xi)+w_{3}(\xi)+w_{2}(\xi) w_{1}(\xi)$ (cf. p. 497, [6] or p. 32 [17]). Thus

$$
\begin{aligned}
w\left(\xi_{1}\right) \cdot & w\left(\lambda^{2}\left(\xi_{2}\right)\right) \\
= & \left(1+w_{2}\left(\xi_{1}\right)+w_{3}\left(\xi_{1}\right)\right) \\
& \times\left(1+\left(w_{1}\left(\xi_{2}\right)\right)^{2}+w_{2}\left(\xi_{2}\right)+w_{3}\left(\xi_{2}\right)+w_{2}\left(\xi_{2}\right) w_{1}\left(\xi_{2}\right)\right) .
\end{aligned}
$$

By Theorem 11.1 [5], the only relations among the Stiefel-Whitney classes of $\xi_{1}, \xi_{2}, \xi_{3}$ over $G(3,3,1)$ are those arising from the single inhomogeneous relation

$$
\begin{aligned}
(1+ & \left.w_{1}\left(\xi_{1}\right)+w_{2}\left(\xi_{1}\right)+w_{3}\left(\xi_{1}\right)\right) \\
& \times\left(1+w_{1}\left(\xi_{2}\right)+w_{2}\left(\xi_{2}\right)+w_{3}\left(\xi_{2}\right)\right)\left(1+w_{1}\left(\xi_{3}\right)\right)=1 .
\end{aligned}
$$

Using the Gysin sequence associated to the double covering $G(3 \mid 3,1) \rightarrow$ $G(3,3,1)$ one can then prove (cf. $\S 15,[17])$ that essentially the same holds in $G(3 \mid 3,1)$, i.e.,

$$
\left(1+w_{2}\left(\xi_{1}\right)+w_{3}\left(\xi_{1}\right)\right) \cdot\left(1+w_{1}\left(\xi_{2}\right)+w_{2}\left(\xi_{2}\right)+w_{3}\left(\xi_{2}\right)\right)\left(1+w_{1}\left(\xi_{3}\right)\right)=1
$$


generates all the relations among the Stiefel-Whitney classes of $\xi_{1}, \xi_{2}, \xi_{3}$ over $G(3 \mid 3,1)$ in $H^{*}\left(G(3 \mid 3,1) ; \mathbf{Z}_{2}\right)$. From this and 4.2 .1 one verifies that

$$
w\left(\xi_{1}\right) \cdot w\left(\lambda^{2}\left(\xi_{2}\right)\right) \neq 1 .
$$

4.3. REMARKs. In concluding, Theorems 1.1(B), (C), and (D) still leave a few unsolved cases. These were listed in 1.2. Attempts by the authors to settle these using the methods of this paper, Stiefel-Whitney classes, or the Kervaire semicharacteristic have all failed.

\section{REFERENCES}

[1] J. F. Adams, Vector fields on spheres, Ann. Math., (3) 75 (1962), 603-632.

[2] E. Antoniano, Sobre las variedades de Stiefel projectivas, Tesis, CIEA del IPN, Mexico, D. F. (1976).

[3] E. Antoniano, S. Gitler, J. Ucci, and P. Zvengrowski, On the K-theory and parallelizability of projective Stiefel manifolds, preprint.

[4] V. Bartík, and J. Korbaš, Stiefel-Whitney characteristic classes and parallelizability of Grassmann manifolds, Proceedings of the 12th Winter School on Abstract Analysis, Supplemento ai Rendiconti del Circolo Mathematico di Palermo II-6-(1984).

[5] A. Borel, La cohomologie mod 2 de certains espaces homogènes, Comm. Math. Helv., 27 (1953), 165-197.

[6] A. Borel and F. Hirzebruch, Characteristic classes and homogeneous spaces-I, Amer. J. Math., 80 (1958), 458-535.

[7] G. E. Bredon and A. Kosinski, Vector fields on m-manifolds, Ann. Math., 84 (1966), 85-90.

[8] M. Fujii, KO-groups of projective spaces, Osaka J. Math., 4 (1967), 141-149.

[9] S. G. Hoggar, On KO-theory of Grassmannians, Quart. J. Math. Oxford (2), 20 (1969), 447-463.

[10] D. Husemoller, Fibre Bundles, 2nd Ed. (1975) Springer-Verlag. GTM-20.

[11] M. Kervaire, Non-parallelizability of the $n$-sphere for $n>7$, Proc. Nat. Acad. Sci., U.S.A. 44 (1958), 504-537.

[12] J. Korbaš, Vector fields on real flag manifolds, Ann. Global Anal. Geom., 3 (1985).

[13] K. Y. Lam, A formula for the tangent bundle of flag manifolds and related manifolds, Trans. Amer. Math. Soc., 213 (1975), 305-314.

[14] M. L. Leite and I. D. Miatello, Linear vector fields on $\tilde{G}_{n, k}$, Proc. Amer. Math. Soc., 80 (1980), 673-677.

[15] I. D. Miatello and R. J. Miatello, On stable parallelizability of $\tilde{G}_{n, k}$ and related manifolds, Math. Ann., 259 (1982), 343-350.

[16] J. Milnor, Some consequences of a theorem of Bott, Ann. Math., (2) 68 (1958), 444-449.

[17] P. Sankaran, Vector fields on flag manifolds, Ph.D. Thesis, The University of Calgary, Calgary, Alberta (1985).

[18] P. Sankaran and P. Zvengrowski, On stable parallelizability of flag manifolds, Pacific J. Math., 122 (1986), 455-458.

[19] N. Steenrod, Topology of Fibre Bundles, Princeton Univ. Press, Princeton, N. J. (1951). 
[20] R. Stong, Semicharacteristics of oriented Grassmannians, J. Pure Appl. Algebra, 33 (1984), 97-103.

[21] W. Sutherland, A note on the parallelizability of sphere bundles over spheres, J. London Math. Soc., 39 (1964), 55-62.

[22] S. Trew and P. Zvengrowski, Non-parallelizability of Grassmann manifolds, Canad. Math. Bull. (1), 27 (1984), 127-128.

[23] P. Zvengrowski, Über die parallelisierbarkeit von Stiefel Mannifaltigkeiten, Forschungsinstitut für Mathematik, ETH Zürich und University of Calgary, April 1976.

Received January 2, 1986. Both authors are indebted to the National Research Council of Canada for financial assistance during this work.

THE UNIVERSITY OF CALGARY

Calgary, Alberta, Canada 



\title{
PACIFIC JOURNAL OF MATHEMATICS EDITORS
}

\author{
V. S. VARADARAJAN \\ (Managing Editor) \\ University of California \\ Los Angeles, CA 90024 \\ HERBERT Clemens \\ University of Utah \\ Salt Lake City, UT 84112 \\ R. FINN \\ Stanford University \\ Stanford, CA 94305
}

\author{
HERMANN FLASCHKA \\ University of Arizona \\ Tucson, AZ 85721 \\ RAMESH A. GANGOLLI \\ University of Washington \\ Seattle, WA 98195 \\ VAUGHAN F. R. JONES \\ University of California \\ Berkeley, CA 94720 \\ ROBION KIRBY \\ University of California \\ Berkeley, CA 94720
}

\author{
C. C. Moore \\ University of California \\ Berkeley, CA 94720
}

H. SAMELSON

Stanford University

Stanford, CA 94305

HAROLD STARK

University of California, San Diego

La Jolla, CA 92093

\section{ASSOCIATE EDITORS}

\author{
R. ARENS \\ E. F. BECKENBACH \\ B. H. NEUMANN \\ F. WOLF \\ K. YOSHIDA \\ (1906-1982)

\section{SUPPORTING INSTITUTIONS} \\ UNIVERSITY OF ARIZONA \\ UNIVERSITY OF BRITISH COLUMBIA \\ CALIFORNIA INSTITUTE OF TECHNOLOGY \\ UNIVERSITY OF CALIFORNIA \\ MONTANA STATE UNIVERSITY \\ UNIVERSITY OF NEVADA, RENO \\ NEW MEXICO STATE UNIVERSITY \\ OREGON STATE UNIVERSITY

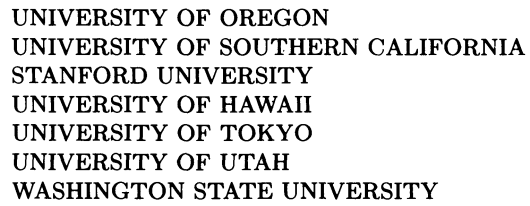

The Supporting Institutions listed above contribute to the cost of publication of this Journal, but they are not owners or publishers and have no responsibility for its content or policies.

Mathematical papers intended for publication in the Pacific Journal of Mathematics should be in typed form or offset-reproduced (not dittoed), double spaced with large margins. Please do not use built up fractions in the text of the manuscript. However, you may use them in the displayed equations. Underline Greek letters in red, German in green, and script in blue. The first paragraph must be capable of being used separately as a synopsis of the entire paper. In particular it should contain no bibliographic references. Please propose a heading for the odd numbered pages of less than 35 characters. Manuscripts, in triplicate, may be sent to any one of the editors. Please classify according to the scheme of Math. Reviews, Index to Vol. 39. Supply name and address of author to whom proofs should be sent. All other communications should be addressed to the managing editor, or Elaine Barth, University of California, Los Angeles, California 90024.

There are page-charges associated with articles appearing in the Pacific Journal of Mathematics. These charges are expected to be paid by the author's University, Government Agency or Company. If the author or authors do not have access to such Institutional support these charges are waived. Single authors will receive 50 free reprints; joint authors will receive a total of $\mathbf{1 0 0}$ free reprints. Additional copies may be obtained at cost in multiples of 50 .

The Pacific Journal of Mathematics is issued monthly as of January 1966. Regular subscription rate: $\$ 190.00$ a year (5 Vols., 10 issues). Special rate: $\$ 95.00$ a year to individual members of supporting institutions.

Subscriptions, orders for numbers issued in the last three calendar years, and changes of address should be sent to Pacific Journal of Mathematics, P.O. Box 969, Carmel Valley, CA 93924, U.S.A. Old back numbers obtainable from Kraus Periodicals Co., Route 100, Millwood, NY 10546.

The Pacific Journal of Mathematics at P.O. Box 969, Carmel Valley, CA 93924 (ISSN 0030-8730) publishes 5 volumes per year. Application to mail at Second-class postage rates is pending at Carmel Valley, California, and additional mailing offices. Postmaster: send address changes to Pacific Journal of Mathematics, P.O. Box 969, Carmel Valley, CA 93924.

PUBLISHED BY PACIFIC JOURNAL OF MATHEMATICS, A NON-PROFIT CORPORATION Copyright (C) 1987 by Pacific Journal of Mathematics 


\section{Pacific Journal of Mathematics}

Vol. 128 , No. $2 \quad$ April, 1987

Pierre Barrucand, John Harold Loxton and Hugh C. Williams, Some

explicit upper bounds on the class number and regulator of a cubic field

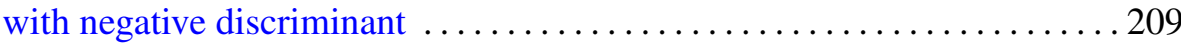

Thomas Ashland Chapman, Piecewise linear fibrations $\ldots \ldots \ldots \ldots \ldots 223$

Yves Félix and Jean-Claude Thomas, Extended Adams-Hilton's

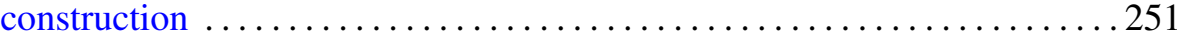

Robert Fitzgerald, Derivation algebras of finitely generated Witt rings . . . 265

K. Gopalsamy, Oscillatory properties of systems of first order linear delay differential inequalities ................................ 299

John P. Holmes, One parameter subsemigroups in locally complete

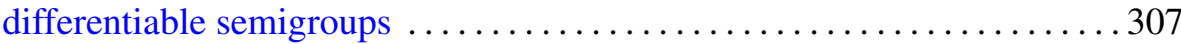

Douglas Murray Pickrell, Decomposition of regular representations for

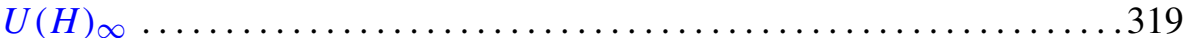

Victoria Powers, Characterizing reduced Witt rings of higher level

Parameswaran Sankaran and Peter Zvengrowski, Stable parallelizability

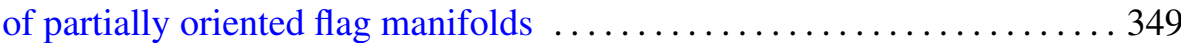

Johan Tysk, Eigenvalue estimates with applications to minimal surfaces . . . 361 Akihito Uchiyama, On McConnell's inequality for functionals of subharmonic functions

Minato Yasuo, Bott maps and the complex projective plane: a construction

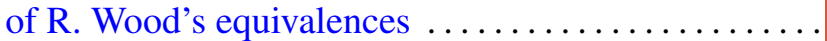

James Juei-Chin Yeh, Uniqueness of strong solutions to stochastic differential equations in the plane with deterministic boundary process 